\section{COMPARISON OF TRANSPORT PATHWAYS OF CATECHOL-SUBSTITUTED \\ CEPHALOSPORINS, BO-1236 AND \\ BO-1341, THROUGH THE \\ OUTER MEMBRANE OF ESCHERICHIA COLI}

\author{
Terutaka Hashizume, Minoru Sanada, \\ Susumu Nakagawa and Nobuo TaNaka \\ Okazaki Research Laboratories, \\ Banyu Pharmaceutical Co., Ltd., \\ 3-9-1 Kamimutsuna, Okazaki 444, Japan
}

(Received for publication June 27, 1990)

The penetration of $\beta$-lactam antibiotics through the outer membrane is a prerequisite for exhibiting the antibacterial activity against Gram-negative bacteria. The elucidation of penetration routes has been the subject of many papers, and our understanding of the functions of a variety of outer membrane proteins has increased in recent years. It is known that major outer membrane proteins which form porin channels, $\mathrm{OmpF}$ and $\mathrm{OmpC}$, allow small hydrophilic molecules including $\beta$-lactam antibiotics to diffuse into the periplasmic space ${ }^{1)}$. On the other hand, catechol-substituted $\beta$-lactam antibiotics, such as BO-1236 ${ }^{2)}$, BO-1341 ${ }^{3,4)}$, E-0702 ${ }^{5)}, \mathrm{M} 14659^{6}$, and pirazmonam ${ }^{7)}$ which all have potent antipseudomonal activity utilize a unique transport pathway, the ton $B$-dependent iron transport pathway in Escherichia coli $^{8)}$. The uptake of iron in $E$. coli is known to require receptor proteins and the ton $B$ gene product ${ }^{9)}$ in conjunction with siderophores. Recently CurTis et al. ${ }^{10}$ ), and NiKaIDo and ROSENBERG ${ }^{11)}$ identified the iron-regulated outer membrane receptor proteins, Fiu and $\mathrm{Cir}$, specific to the catechol-substituted cephalosporins.

In our paper, we report the diversity of the transport pathway as assessed from the characterization of spontaneous mutants of $E$. coli selected with BO-1236 and BO-1341. We also note the influence of bacterial growth under anaerobic condition and in the presence of ascorbic acid as well as iron concentration in the medium, on the antibacterial activity of these catechol-substituted cephalosporins.

BO-1236 and BO-1341, both of which were cephem antibiotics as shown in Fig. 1, were synthesized at the Okazaki Research Laboratories of Banyu Pharmaceutical Co., Ltd. (Okazaki, Japan). Ceftazidime, and cefoxitin were commercial products of Glaxo Japan Co, Ltd. (Tokyo, Japan), and Banyu Pharmaceutical Co., Ltd. (Tokyo, Japan), respectively. The strains used in this study were $E$. coli $\mathrm{CS} 109, E$. coli $\mathrm{K}-12$ substrain, and $E$. coli $\mathrm{CS} 197$, an OmpF and OmpC deficient mutant derived from $\mathrm{CS} 109^{12)}$. The mutants resistant to BO-1236 and BO-1341 were spontaneously isolated on the antibiotic-containing agar plates. The culture media used were: Mueller-Hinton agar (Difco) for the isolation of mutants and the determination of MIC, and M9 medium ${ }^{13)}$ containing $0.2 \%$ Casamino Acids without iron chelators for the outer membrane preparation. Cells diluted to $10^{6} \mathrm{cfu} / \mathrm{ml}$ were inoculated onto the antibiotic-containing agar plates for the MIC determination. The outer membranes were prepared by differential centrifugation, and treatment with sodium sarcosinate ${ }^{14)}$. The resulting preparations were used for the analysis of outer membrane proteins with the SDS/PAGE system described by LUNDRIGAN and EARHART ${ }^{15)}$.

The spontaneous mutants resistant to BO-1236 and $\mathrm{BO}-1341$ were isolated from colonies of the parent strain, CS109, grown on agar plates containing the highest concentration of BO-1236 and $\mathrm{BO}-1341$ by spreading the overnight culture. Prior to selection of a mutant, approximately ten colonies randomly picked out were confirmed to have similar resistant levels against the antibiotics tested. Thus the two mutants of E. coli CS109, BB5936 and BB5937 were selected on the agar plates containing 16-fold-greater MIC of BO-1236 (isolation frequency: approximately $7 \times 10^{-8}$ ) and 64fold greater MIC of BO-1341 (isolation frequency: approximately $4 \times 10^{-7}$ ), respectively. As shown in Table 1, the strain BB5936, with the 8-fold greater MIC of BO-1236 by the conventional method of

Fig. 1. Chemical structures of BO-1236 and BO-1341<smiles>CC(C)(O/N=C(/C(=O)O)c1csc(N)n1)OC(=O)C(=O)[O-]</smiles>

BO-1236<smiles>[R]C[N+]1(C)Cc2cc(O)c(O)cc2C1</smiles><smiles></smiles> 
Table 1. Susceptibility of Escherichia coli mutants selected with BO-1236 and BO-1341.

\begin{tabular}{llcccc}
\hline & \multicolumn{1}{c}{ Characteristics } & \multicolumn{2}{c}{ MIC $(\mu \mathrm{g} / \mathrm{ml})^{b}$} \\
\cline { 3 - 6 } Strain $^{\mathrm{a}}$ & & BO-1236 & BO-1341 & Ceftazidime & Cefoxitin \\
\hline CS109 & Wild & 0.05 & 0.013 & 0.1 & 3.13 \\
BB5936 & envZ mutant & 0.39 & 0.1 & 0.39 & 12.5 \\
BB5937 & ton $B$ mutant & 0.78 & 3.13 & 0.1 & 3.13 \\
BB5938 & envZ tonB double mutant & 6.25 & 25 & 0.39 & 12.5 \\
CS197 & OmpF $^{-}$, OmpC $^{-}$ & 0.05 & 0.013 & 0.39 & 25 \\
\hline
\end{tabular}

a See the text.

b Determined by agar dilution method with Mueller-Hinton agar (Difco).

MIC determination, exhibited relatively low resistance to ceftazidime and cefoxitin as well as BO-1236 and BO-1341, compared with the susceptibility levels of the parent strain, CS109. The strain BB5937, having the 256-fold greater MIC of BO-1341, was identified as a ton $B$ mutant, as previously reported ${ }^{2)}$. The ton $B$ mutant apparently acquired the moderate resistance specific to BO-1236 and BO-1341 as indicated by the higher MICs than those for the strain BB5936. The strain BB5938, which was derived from the strain BB5937 with further exposure to 2-fold greater MIC of BO1341 (isolation frequency: approximately $1 \times 10^{-7}$ ), showed much higher resistance to both $\mathrm{BO}-1236$ and BO-1341 than did the $\operatorname{ton} B$ mutant, and low resistance to ceftazidime and cefoxitin. The $\mathrm{OmpF}$ and OmpC deficient mutant strain (CS197) showed resistance to ceftazidime and cefoxitin, but was fully susceptible to BO-1236 and BO-1341.

The profiles of outer membrane proteins of the mutants was shown in Fig. 2. There were no appreciable differences in the $\mathrm{OmpF}$ and $\mathrm{OmpC}$ proteins between wild-type strain, CS109, and the tonB mutant, BB5937, while the strain CS197 was devoid of these porin proteins. The strains of BB5936 and BB5938, having similar outer membrane profiles to each other, were deficient in $\mathrm{OmpF}$ protein, protein $\mathrm{a}$, and the iron-regulated outer membrane proteins (Fiu, 83 kilodaltons ( $\mathrm{kDa}$ ); FepA, $81 \mathrm{kDa}$; $\mathrm{Cir}, 74 \mathrm{kDa}$ ), and overproduced OmpC protein. These profiles phenotypically agreed with the description of the env $Z$ (perA) mutant reported by LUNDRIGAN and EARHART ${ }^{15)}$, and the resistance to $\lambda$ phage also supported the evidence of mutation at env $Z$ locus. The deficiency of the OmpF protein reduced the susceptibility to ceftazidime and cefoxitin (Table 1) ${ }^{16}$. Hence, the strains (BB5936 and BB5938) were assigned to be $e n v Z$ mutant, and env $Z$ ton $B$ mutant, respectively.

In this study, we found the reduced levels of their
Fig. 2. Comparison of outer membrane proteins of the strains spontaneously isolated mutants resistant to BO-1236 and BO-1341.

Strains were: (A), CS109 (wild-type); (B), BB5936; (C), BB5937; (D), BB5938 and (E), CS197 (OmpF ${ }^{-}$, $\mathrm{OmpC}^{-}$).

(A) (B) (C) (D) (E)

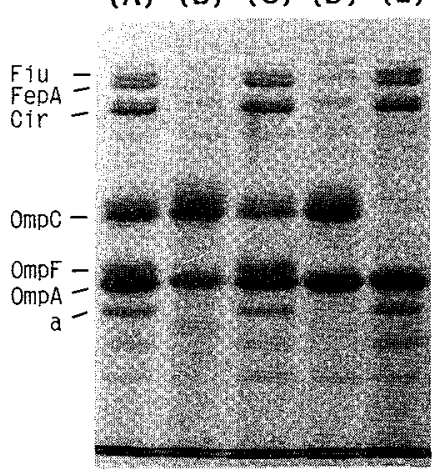

The $25 \mu \mathrm{g}$ of each protein was run with the $8 \mathrm{M}$ urea-containing SDS/PAGE described by LUNDRIGAN and EARHART ${ }^{15)}$.

possible receptor proteins, $\mathrm{Fiu}$ and $\mathrm{Cir}$, as a result of envZ mutation (Fig. 2). However, the exclusive fiu cir mutant was not isolated probably due to its extremely low isolation frequency of double mutant. The tonB mutant (BB5937) was 8-fold and 256-fold less susceptible than the wild-type strain to $\mathrm{BO}-1236$ and BO-1341, respectively. The envZ tonB mutant, BB5938, spontaneously derived from the $\operatorname{ton} B$ mutant, BB5937, also showed the further 8-fold increase in the resistance to both BO-1236 and BO-1341, indicating that the both compounds diffuse through the $\mathrm{OmpF}$ channel as well as the ton $B$-dependent iron transport pathway.

We previously reported that the $\operatorname{ton} B$ mutant was selected with BO-1341 based on analysis using relevant mutants and phages ${ }^{3)}$, so that the cephem penetrates via the $\operatorname{ton} B$-dependent iron transport 
Table 2. Susceptibility of Escherichia coli CS109 under the various conditions.

\begin{tabular}{lcccc}
\hline \multirow{2}{*}{$\begin{array}{c}\text { Culture } \\
\text { condition }\end{array}$} & \multicolumn{4}{c}{ MIC $(\mu \mathrm{g} / \mathrm{ml})$} \\
\cline { 2 - 5 } & BO-1236 & BO-1341 & Ceftazidime & Cefoxitin \\
\hline Control $^{\mathrm{a}}$ & 0.05 & 0.013 & 0.1 & 3.13 \\
$\mathrm{FeCl}_{3}{ }^{\mathrm{b}}$ & 0.39 & 0.2 & 0.1 & 3.13 \\
Anaerobic $^{\mathrm{c}}$ & 0.78 & 6.25 & 0.1 & 3.13 \\
Ascorbic & 0.39 & 0.78 & 0.1 & 3.13 \\
\multicolumn{1}{c}{ acid $^{\mathrm{d}}$} & & & & \\
\hline
\end{tabular}

a Determined by agar dilution method with MuellerHinton agar (Difco).

b Ferric chloride, $1 \mathrm{~mm}$, was added in the medium.

c Cultured under anaerobic condition using GasPak Jar (BBL).

d Ascorbic acid, $0.1 \%(\mathrm{w} / \mathrm{v})$, was added in the medium.

pathway. However, the attempt to isolate spontaneous mutants resistant to BO- 1236 resulted in the env $Z$ mutant. In order to confirm whether BO-1236 also utilizes the ton $B$-dependent iron transport system, the influence of bacterial growth, under the anaerobic condition and in the presence of ascorbic acid as well as ferric iron in the medium, on the antibacterial activity of BO-1236 and BO-1341 was investigated (Table 2). Although the degree of reduction in susceptibility to BO-1236 was smaller than that to BO-1341, the susceptibilities to both antibiotics were reduced under anaerobic condition and in the presence of ascorbic acid as well as ferric iron. In contrast, the susceptibilities to ceftazidime and cefoxitin were not influenced under these conditions. The result reflected the suppression of ton $B$-dependent ferric iron uptake system under these conditions ${ }^{17)}$, and $\mathrm{BO}-1236$ seemed to penetrate the outer membrane through the ton $B$ dependent iron transport pathway as did BO-1341.

The env $Z$ mutant selected with BO-1236 showed the reduced levels of the iron-regulated outer membrane proteins, and OmpF protein (Fig. 2) that are responsible for the resistance to catecholsubstituted cephalosporins and the cephalosporins like ceftazidime and cefoxitin ${ }^{15)}$, respectively. The unaltered susceptibility of CS109 and CS197, the OmpF and OmpC deficient mutant, to BO-1236 and BO-1341 was attributed to the efficient penetration through the ton $B$ pathway, even if $\mathrm{OmpF}$ and $\mathrm{OmpC}$ were not available.

The tonB mutant (BB5937) showed higher increase in resistance to $\mathrm{BO}-1341$ than to $\mathrm{BO}-1236$ (Table 1). It is likely that BO-1341 largely depends on the ton $B$-dependent iron transport pathway for the penetration route, while BO-1236 does not utilize the $\operatorname{ton} B$-dependent iron transport pathway so much as BO-1341. Taking into consideration that the wild-type strain does not produce detectable $\beta$-lactamase, and both cephalosporins have similar affinity for PBPs 1A, 1B and 3 of $E$. coli (data not shown), it is suggested the ton $B$-dependent transport pathway is more effective than porin channels for these cephalosporins, because of the 4-fold higher activity of BO-1341 than that of BO-1236 against the strain. In contrast, $\mathrm{BO}-1236$ has the merits that the susceptibility to BO-1236 was less affected by $\operatorname{ton} B$ mutation than that to $\mathrm{BO}-1341$, and the frequency of emergence of spontaneous mutant resistant to BO-1236 was not only lower than that to BO-1341, but also a ton $B$ mutant did not emerge.

We showed the possible development of spontaneous resistance to catechol-substituted cephalosporins in patients. The ton $B$ mutant seems to be nonpathogenic due to the growth defect under iron-deficient in vivo environment ${ }^{6,10)}$, and we also found that the env $Z$ mutant with lower resistance was even more susceptible to both cephalosporins under iron-deficient medium (data not shown). However, our results suggest that facultative anaerobic bacteria including such spontaneous mutants might survive in the infection sites where the bacteria can grow anaerobically, because the ton $B$-dependent transport pathway is mainly exploited by the catechol-substituted cephalosporins under aerobic condition.

Nevertheless, the utilization of tonB-dependent transport pathway besides porin channels allows the effective penetration of antibiotics to periplasm of bacteria which have low outer membrane permeability like $P$ seudomonas aeruginosa ${ }^{2 \sim 4)}$.

\section{Acknowledgments}

We thank N. HAZUMI for testing $\lambda$ phage sensitivity, and $\mathrm{K}$. ImaI for excellent technical assistance.

\section{References}

1) NiKaIDO, H. \& E. Y. RosenBERG: Effect of solute size on diffusion rates through the transmembrane pores of the outer membrane of $E$. coli. J. Gen. Physiol. 77: $121 \sim 135,1981$

2) Nakagawa, S.; M. Sanada, K. Matsuda, N. HAZUMI \& N. TANAKA: Biological activity of BO-1236, a new antipseudomonal cephalosporin. Antimicrob. Agents Chemother. 31: 1100 1105, 1987

3) Sanada, M.; T. Hashizume, K. Matsuda, S. NAKAGAWA \& N. TANAKA: Mode of action of 
BO-1341: Transport pathway through the outer membrane of Escherichia coli. Drugs. Exp. Clin. Res. 14: $397 \sim 402,1988$

4) Nakagawa, S.; M. Sanada, K. Matsuda, T. Hashizume, Y. Asaht, R. Ushijma, N. Ohtake \& N. TANAKA: In vitro and in vivo antibacterial activities of BO-1341, a new antipseudomonal cephalosporin. Antimicrob. Agents Chemother. 33: $1423 \sim 1427,1989$

5) Watanabe, N.; T. Nagasu, K. Katsu \& K. KitoH: $\mathrm{E}-0702$, a new cephalosporin, is incorporated into Escherichia coli cells via the tonB-dependent iron transport system. Antimicrob. Agents Chemother. 31: $497 \sim 504,1987$

6) Mochizuki, H.; Y. Oikawa, H. Yamada, S. Kusakabe, T. Shihara, K. Murakam, K. Kato, J. IsHiguro \& $\mathrm{H}$. KosuzumE: Antibacterial and pharmacokinetic properties of $\mathrm{M} 14659$, a new injectable semisynthetic cephalosporin. J. Antibiotics 41: $377 \sim 391,1988$

7) Bush, K.; S. K. Tanaka, S. Ohringer \& D. P. BonNer: Mode of action studies: Pirazmonam in Escherichia coli and Pseudomonas aeruginosa. Program and Abstracts of the 27th Intersci. Conf. on Antimicrob. Agents Chemother., No. 1218, p. 309, New York, Oct. 4 7, 1987

8) Braun, V.; R. E. W. Hancock, K. Hantke, \& A. HARTMAN: Functional organization of the outer membrane of Escherichia coli: Phages and colicin receptors as components of iron uptake system. J. Supramol. Struct. 5: $37 \sim 58,1976$

9) BAGG, A. \& J. B. NEILANDS: Molecular mechanism of regulation of siderophore-mediated iron assimilation. Microbiol. Rev. 51: 509 518, 1987
10) Curtis, N. A. C.; R. L. Eisenstadt, S. J. East, R. J. Cornford, L. A. WAlker \& A. J. White: Ironregulated outer membrane proteins of Escherichia coli $\mathrm{K}-12$ and mechanism of action of catecholsubstituted cephalosporins. Antimicrob. Agents Chemother. 32: $1879 \sim 1886,1988$

11) Nikaido, H. \& E. Y. Rosenberg: Cir and Fiu proteins in the outer membrane of Escherichia coli catalyze transport of monomeric catechols: Study with $\beta$-lactam antibiotics containing catechol and analogous groups. J. Bacteriol. 172: $1361 \sim 1367,1990$

12) Pugsley, A. P. \& C. A. Schnattman: Outer membrane proteins of Escherichia coli. VII. Evidence that bacteriophage-directed protein 2 functions as a pore. J. Bacteriol. 133: 1181 1189, 1978

13) Miller, J. H. (Ed.): Experiments in Molecular Genetics. Cold Spring Harbor Laboratory, 1972

14) Sawai, T.; R. Hiruma, N. Kawana, M. Kaneko, F. TANIYASU \& A. INAMI: Outer membrane permeation of $\beta$-lactam antibiotics in Escherichia coli, Proteus mirabilis, and Enterobacter cloacae. Antimicrob. Agents Chemother. 22: 585 592, 1982

15) LUNDRIGAN; M. \& C. F. EARHART: Reduction in three iron-regulated outer membrane proteins and protein a by the Escherichia coli K-12 perA mutantion. J. Bacteriol. 146: 804 807, 1981

16) JAfFé, A.; Y. A. Chabbert \& E. Derlot: Selection and characterization of $\beta$-lactam-resistant Escherichia coli K-12 mutants. Antimicrob. Agents Chemother. 23: 622 625, 1983

17) HANTKE, K.: Regulation of ferric iron transport in Escherichia coli K12: Isolation of a constitutive mutant. Mol. Gen. Genet. 182: 288 292, 1981 